\title{
Using Symmetry to Improve Percolation Threshold Bounds
}

\author{
William D. May and John C. Wierman \\ Department of Mathematical Sciences \\ Johns Hopkins University
}

November 17, 2003

\begin{abstract}
We show that symmetry, represented by a graph's automorphism group, can be used to greatly reduce the computational work for the substitution method. This allows application of the substitution method over larger regions of the problem lattices, resulting in tighter bounds on the percolation threshold $p_{c}$. We demonstrate the symmetry reduction technique using bond percolation on the $\left(3,12^{2}\right)$ lattice, where we improve the bounds on $p_{c}$ from $(0.738598,0.744900)$ to $(0.739399,0.741757)$, a reduction of more than $62 \%$ in width, from 0.006302 to 0.002358 .
\end{abstract}

\section{Introduction}

Percolation processes were introduced in 1957 by Broadbent and Hammersley [3] as models for the flow of fluid through a random medium. A Bernoulli bond percolation model is comprised of an infinite lattice graph $\mathcal{G}$, with each bond (that is, edge) independently designated as open with probability $p$, $0<p<1$, and closed otherwise. The open cluster containing a specific vertex $v \in \mathcal{G}$, denoted $C_{v}$, is the set of all vertices that can be reached from $v$ through a path of open bonds. Let $P_{p}^{\mathcal{G}}$ denote the probability measure corresponding to probability $p$. An important quantity is the percolation probability $\theta_{v}^{\mathcal{G}}(p)=P_{p}^{\mathcal{G}}\left(\left|C_{v}\right|=\infty\right)$. The critical probability, also called the percolation threshold, of the lattice is then defined by $p_{c}(\mathcal{G})=\inf \left\{p: \theta_{v}^{\mathcal{G}}(p)>0\right\}$, which is independent of the vertex $v$ if $\mathcal{G}$ is connected.

One goal of percolation theory is to understand the dependence of the critical probability on the properties of a lattice graph, and to find accurate approximation formulas based on these properties. To develop such approximations we first need to know the critical probabilities for a variety of lattices. This has proven to be a difficult problem. After almost 40 years of effort, exact critical probability values are known for only a handful of lattices, and the published approximations for other lattices are either loose bounds or not rigorously obtained (for example, from simulations).

The substitution method was introduced by Wierman [15] as a technique for rigorously determining bounds on $p_{c}(\mathcal{G})$ for many two-dimensional bond and site percolation models. Wierman describes several recent results obtained using the substitution method in [16], [17], and [18] .

Unfortunately, there are computational difficulties when applying the method over large portions of the lattice, so the bounds obtained are often not tight enough to either reject or strongly support conjectured critical probability values, or to be competitive with simulation estimates. In this paper we introduce a computational technique that allows us to apply the substitution method to a larger subgraph of the lattice, thus obtaining tighter bounds on the critical probability. The basis of the improvement lies in exploiting symmetry of the finite subgraph $G$ used as the substitution region. This symmetry is 
encapsulated in the automorphism group of $G$. When we apply the automorphism group action to the partially ordered set (poset) that describes the connectivity of the subgraph, we reduce the original poset to a much smaller poset. The substitution method calls for finding the extremal solutions for polynomial equations derived from up-sets of the original, large, partially ordered set. We will show that we obtain the same solutions by searching the symmetry reduced poset. This makes the algorithm tractable for larger regions of the unsolved lattice, so that we can obtain tighter bounds on $p_{c}$.

The improvement due to the results of this paper is beyond that achievable by improvements in computing power alone. In the illustrative example of Section 5, there is six-fold symmetry, which reduces the size of the poset from 203 to 37 elements. The number of up-sets is reduced from greater than $10^{27}$ to 95,708 . Thus the reduction of the size of the poset by a factor of around 6 reduces the number of up-sets by roughly the sixth root.

Note that symmetry was used in a less systematic fashion in Wierman [17] and [19]. Due to the results of this paper, the detailed justifications in Section 4 of [17] and Section 4 of [19] are no longer necessary.

This paper is organized as follows. In Section 2 we review the substitution method, its theoretical foundation, and the definitions and notation used throughout this paper. In Section 3 we show how the automorphism group of the substitution region subgraph can be used to reduce the partition lattice to the much smaller class lattice. Section 4 contains our main theoretical result, where we show that the extremal up-set equation solutions on the class lattice equal the extremal solutions on the partition lattice. Due to the reduced size of the lattice these solutions can be found with much less work.

In Section 5 we use the improved algorithm to calculate bounds on $p_{c}$ for the $\left(3,12^{2}\right)$ lattice. Our calculation tightens the rigorously obtained bounds (see Wierman [18]) from $(0.738598,0.744900)$ to $(0.739399,0.741757)$. There is a longstanding conjecture by Tsallis [13] that the exact percolation threshold for this lattice is $.739830 \ldots$... While our rigorous bounds do not reject this value, the trend of lower bounds suggests that Tsallis's conjecture is too low and may be disproved when we apply the substitution method to a sufficiently large region.

Work by other researchers also casts doubt on Tsallis's conjecture. Tsallis used the same technique to calculate conjectured thresholds for both the $\left(3,12^{2}\right)$ and Kagomé lattices $(.739830 \ldots$ and $0.522372 \ldots$, respectively). Using the hull-gradient method, Ziff and Suding [20] find a $p_{c}$ value of $0.5244053 \pm$ 0.0000003 for the Kagomé lattice. Jensen et. al. [4] provide further evidence (not proof) that the value conjectured by Tsallis is too low for the Kagomé lattice. Thus there is accumulating evidence that Tsallis's technique produces $p_{c}$ values that are too low.

Finally, in an appendix we show how to calculate the number of elements in the symmetry reduced poset. This calculation is needed to verify results in the computer program, as well as being an interesting problem in its own right.

\section{The Substitution Method}

The substitution method was introduced by Wierman [15] to find bounds for the critical probabilities of bond percolation models in two dimensions. It allows one to derive information by comparing the percolative behavior of two different lattices at different parameter values. Typically the unsolved lattice is compared to a reference lattice with exactly known critical probability, in order to gain information about the unsolved case.

Consider an infinite lattice $\mathcal{G}$ that may be decomposed into a union of isomorphic, edge-disjoint, finite, connected subgraphs in such a way that every edge is in a subgraph and every vertex is in at least one subgraph. Vertices that are in more than one subgraph are called boundary vertices. A path on the lattice may enter or leave the subgraph in the decomposition only through its boundary vertices.

Now consider a fixed subgraph $G$ in this decomposition. Denote its boundary vertices by $A, B, C, \ldots$. Any designation of edges as open or closed on $G$ determines a partition of the boundary vertices into clusters of vertices that are connected by open edges. Each such boundary partition, or simply partition, may be denoted by a sequence of vertices separated by vertical bars, where vertices are in distinct open clusters if and only if they are separated by a vertical bar. For example, if the boundary vertex set consists of $A, B$, and $C$ then $A \mid B C$ indicates that $B$ and $C$ are in the same open cluster, but $A$ is in a different 
cluster.

Given the boundary vertices, we use $\mathcal{B}$ to represent the set of all partitions of the set $A, B, C, \ldots$ A partition of $A, B, C, \ldots$ is in $\mathcal{B}$ regardless of whether or not there is a collection of open and closed edges in $G$ that give rise that partition.

A partition $\pi$ is a refinement of partition $\sigma$, denoted $\pi \leq \sigma$, if every cluster of $\pi$ is contained entirely in a cluster of $\sigma$. The set of boundary partitions with refinement form a partially ordered set, called the partition lattice $\mathcal{P}$. (It is a lattice because all pairs of elements have a least upper bound and a greatest lower bound). $\sigma$ is said to cover $\pi$ if $\pi<\sigma$ and there is no element $\xi \in \mathcal{P}$ satisfying $\pi<\xi<\sigma$. We write $\mathcal{P}_{n}$ to indicate the partition lattice with $n$ boundary vertices.

We will use some standard terminology from the literature on partially ordered sets (see Stanley [9]). The maximal element, $\hat{1}$, is the partition with all boundary vertices connected, while the minimal element, $\hat{0}$, has each boundary vertex in a distinct cluster. A chain is a subposet of $\mathcal{P}$ in which any two elements are comparable. In contrast, an antichain is a subposet of $\mathcal{P}$ in which any two elements are incomparable. The length of a chain $\ell(C)$ is defined $\ell(C)=|C|-1$. The rank of $\mathcal{P}_{n}$ is the maximum length of a chain in $\mathcal{P}_{n}$, which is easily seen to be $n-1$. Every maximal length chain of $\mathcal{P}_{n}$ has the same length, so $\mathcal{P}_{n}$ is said to be graded of rank $n-1$. In this case there is a unique rank function $\rho: \mathcal{P} \rightarrow\{0,1, \ldots, n-1\}$ such that $\rho(x)=0$ if $x$ is the minimal element of $\mathcal{P}_{n}$, and $\rho(y)=\rho(x)+1$ if $y$ covers $x$ in $\mathcal{P}_{n}$. If $\rho(x)=i$ then we say $x$ has rank $i$. For the partition lattice $\mathcal{P}_{n}$ an element of rank $i$ has $n-i$ clusters of boundary vertices. Two posets $\mathcal{P}$ and $\mathcal{Q}$ are said to be isomorphic if there exists an order-preserving bijection $f: \mathcal{P} \rightarrow \mathcal{Q}$ whose inverse is order-preserving, that is:

$$
x \leq y \text { in } \mathcal{P} \Leftrightarrow f(x) \leq f(y) \text { in } \mathcal{Q} .
$$

The Bernoulli bond percolation model on $\mathcal{G}$ with parameter $p$ assigns probability to each assignment of open or closed edges of $G$, which is called a bond configuration. The bond configuration can be identified with a subgraph of $G$ by deleting edges that are closed in the configuration. If $\kappa$ represents a bond configuration, then $V(\kappa)=V(G)$ and $E(\kappa) \subseteq E(G)$, where $V(G)$ and $E(G)$ are the standard notation for the vertex set and edge set of an undirected graph. We write $2^{E(G)}$ for the set of all bond configurations on $G$.

For the Bernoulli bond percolation model the probability polynomial for bond configuration $\kappa$ is

$$
P_{\kappa}^{G}(p)=p^{|E(\kappa)|}(1-p)^{|E(G)|-|E(\kappa)|} .
$$

Probability measures of individual bond configurations are at too fine a level of detail to be useful. More often we are interested in the probability of sets of bond configurations. Since bond configurations are mutually exclusive events, the probability polynomial for a set $S \subseteq 2^{E(G)}$ is the sum of the probabilities of the bond configurations in $S$. Thus we define

$$
P_{S}^{G}(p)=\sum_{\kappa \in S} P_{\kappa}^{G}(p)=\sum_{\kappa \in S} p^{|E(\kappa)|}(1-p)^{|E(G)|-|E(\kappa)|}
$$

to represent the probability polynomial for $S$.

An important set probability polynomial is the boundary partition probability polynomial. Let $\Pi$ : $2^{E(G)} \rightarrow \mathcal{B}$ be the mapping from a bond configuration to its boundary partition. Then the boundary partition probability polynomial, or more simply the partition polynomial, for the boundary partition $\pi$ is

$$
P_{\pi}^{G}(p)=\sum_{\kappa: \Pi(\kappa)=\pi} P_{\kappa}^{G}(p) .
$$

We often write $\pi$-polynomial to refer to a generic boundary partition probability polynomial.

Suppose that another lattice $\mathcal{H}$ may be decomposed into subgraphs with the same number of boundary vertices, corresponding to the substitution of a subgraph for each subgraph in the decomposition of $\mathcal{G}$. Consider the subgraph $H$ in $\mathcal{H}$ substituted for $G$ in $\mathcal{G}$, and identify the boundary vertices with $A, B, C, \ldots$ As above, a probability measure $P_{\pi}^{H}(q)$ on the partition lattice of partitions of $A, B, C, \ldots$ is determined from the percolation model on $\mathcal{H}$ with parameter $q$.

In applying the substitution method to percolation models, the relevant comparison of two probability measures $P_{\bullet}^{G}(p)$ and $P_{\bullet}^{H}(q)$ on the partition lattice is stochastic ordering. An up-set of a partially 
ordered set $\mathcal{P}=(S, \leq)$ is a subset $U \subseteq S$ such that if $g \geq f$ and $f \in U$ then $g \in U$ (note that the empty set is an up-set). If $P$ and $Q$ are two probability measures on $\mathcal{P}$ then we say $P$ is stochastically smaller than $Q$, denoted $P \leq{ }_{s} Q$, if $P[U] \leq Q[U]$ for every up-set $U$.

The key fact is that for probability measures on a finite partially ordered set, stochastic ordering is equivalent to the existence of a coupling. This is a consequence of Strassen's Theorem [11] (or see Lindvall [5]). This means that if $P_{\bullet}^{G}(p) \leq_{s} P_{\bullet}^{H}(q)$, there exist jointly distributed random partitions $X$ and $Y$ such that $X$ is a refinement of $Y$ with probability one, $X$ has marginal distribution $P^{G}(p)$ and $Y$ has marginal distribution $P^{H}(q)$. Note that $X$ represents a realization of the percolation model on $G$ with parameter $p$ and $Y$ represents a realization of the percolation model on $H$ with parameter $q$ such that any pair of boundary vertices that are connected by an open path in $G$ according to $X$ are also connected by an open path in $H$ according to $Y$.

Combining such couplings for all copies of $G$ in the infinite graph $\mathcal{G}$ we obtain coupled realizations of the bond percolation models on $\mathcal{G}$ and $\mathcal{H}$ such that any pair of boundary vertices that are connected by an open path in $\mathcal{G}$ are also connected by an open path in $\mathcal{H}$. Thus the percolation probability values are ordered: $\theta^{\mathcal{G}}(p) \leq \theta^{\mathcal{H}}(q)$. If $\mathcal{H}$ is a reference lattice, and $q_{0}=p_{c}(\mathcal{H})$, we may determine upper and lower bounds for $p_{c}(\mathcal{G})$ by finding $p_{u}=\min \left\{p: P^{G}(p) \geq{ }_{s} P^{H}\left(q_{0}\right)\right\}$ and $p_{l}=\max \left\{p: P^{G}(p) \leq_{s} P^{H}\left(q_{0}\right)\right\}$, respectively.

This characterization of $p_{u}$ and $p_{l}$ may be reinterpreted as follows. We define the up-set probability polynomial for $G$ (and similarly for $H$ ), by

$$
P_{U}^{G}(p)=\sum_{\pi \in U} P_{\pi}^{G}(p)=\sum_{\pi \in U} \sum_{\{\kappa: \Pi(\kappa)=\pi\}} P_{\kappa}^{G}(p),
$$

where $U$ is an up-set. An equivalent means of obtaining $p_{l}$ and $p_{u}$ is to solve for the extremal solutions in $p$ to the up-set equations $P_{U}^{G}(p)=P_{U}^{H}\left(q_{0}\right)$, over all up-sets $U$. Note that $P_{U}^{G}(p)$ is a polynomial and $P_{U}^{H}\left(q_{0}\right)$ is a real number, so the problem reduces to finding roots of univariate polynomials.

\subsection{Properties of the Probability Polynomials}

We establish some properties of the probability polynomials that will be needed in this paper.

$P_{\pi}^{G}(p)$ is the (possibly empty) sum of terms of the form $p^{k}(1-p)^{n-k}$. If for any $p \in(0,1)$ one of these terms is greater than zero, then it is greater than zero for all $p \in(0,1)$, and so is the sum. Therefore:

Fact 1. For any partition $\pi, P_{\pi}^{G}(p)$ is either identically 0 or $P_{\pi}^{G}(p)>0$ for all $p \in(0,1)$.

Up-set polynomials are formed by summing the $\pi$-polynomials of an up-set. Let $P_{U}^{G}(p)$ be an up-set polynomial. Except for the up-set polynomial for the up-set generated by the 0 partition, $P_{U}^{G}(0)=0$, $P_{U}^{G}(1)=1$, and $P_{U}^{G}(p) \geq 0 \forall p \in[0,1]$. For the up-set generated by the $\hat{0}$ partition $P_{U}^{G}(p)=1 \forall p \in$ $[0,1]$.

A standard coupling argument shows that $P_{U}^{G}(p)$ is a nondecreasing function of $p$, but we need a slightly stronger result. $P_{U}^{G}(p)$ is a polynomial onto $[0,1]$. From the Intermediate Value Theorem, we infer that a solution to the up-set equation exists, but need not be unique. Let us assume that there are two solutions to the up-set equation, $a$ and $b$, such that $a<b$ and $P_{U}^{G}(a)=P_{U}^{G}(b)=y$. Since $P_{U}^{G}(p)$ is nondecreasing, it must be constant for $p \in[a, b]$. The only polynomial constant on an interval is constant everywhere. But an up-set polynomial is onto $[0,1]$, so it is not a constant. Thus we see that

Fact 2. Except for the empty up-set and the up-set generated by the disconnected partition, the solution to the up-set equation $P_{U}^{G}(p)=y$ for $y \in[0,1]$ exists and is unique.

\section{Group Actions}

The infinite lattices analyzed using the substitution method are usually derived from tilings of the plane by polygons. In such cases the subgraph employed in the substitution method usually exhibits considerable symmetry. In this section we exploit this symmetry, in the form of the subgraph's automorphism 
group $\operatorname{Aut}(G)$, to reduce the partition lattice to a much smaller partially ordered set that we call the class poset (or more precisely, the class lattice).

\subsection{The Automorphism Group}

An automorphism of a graph $G$ is a permutation of the vertices $f: V(G) \rightarrow V(G)$ with the property that $\{u, v\}$ is an edge of $G$ if and only if $\{f(u), f(v)\}$ is an edge of $G$. In other words, if vertices $u$ and $v$ are adjacent before applying $f$, then their images are adjacent after applying $f$.

A well known fact is that the set of automorphisms with composition form a group (see Biggs [2]). Thus every permutation $f \in \operatorname{Aut}(G)$ has an inverse, and $\operatorname{Aut}(G)$ is closed under composition. Aut $(G)$ is non-empty since the identity permutation satisfies the definition for all graphs.

We establish two facts that will be used in later development. Applying $f$ to a bond configuration $\kappa$ relabels the vertices, which doesn't change the number of open and closed bonds. Therefore the probability polynomial must remain unchanged. Stating this formally:

Fact 3. If $\kappa$ and $f(\kappa)$ are two bond configurations, with $f \in A u t(G)$, then $P_{\kappa}^{G}(p)=P_{f(\kappa)}^{G}(p), \forall p \in$ $[0,1]$.

An automorphism $f$ also relates boundary partitions. $f$ applied to a bond configuration $\kappa_{1}$ is a permutation of the vertices of the graph, resulting in a bond configuration $\kappa_{2}$. Similarly, applying $f$ to the vertex labels in the boundary partition derived from $\kappa_{1}$ (that is, $\pi_{1}$ ) results in the boundary partition for $\kappa_{2}$ (that is, $\pi_{2}$ ). Thus:

Fact 4. Given bond configurations $\kappa_{1}$ and $\kappa_{2}$ such that $\kappa_{2}=f\left(\kappa_{1}\right)$, with $f \in \operatorname{Aut}(G)$. Let $\pi_{1}=\Pi\left(\kappa_{1}\right)$ and $\pi_{2}=\Pi\left(\kappa_{2}\right)$ be the corresponding boundary partitions. Then $\pi_{2}=f\left(\pi_{1}\right)$.

When we apply group actions as part of the substitution method, we may not be able to use $\operatorname{Aut}(G)$ itself. The group we use must satisfy two constraints. First, since we are comparing percolation on two graphs, the unsolved graph $G$ and the reference graph $H$, the group elements must be valid automorphisms for both graphs. More precisely, the group we use must be a subgroup of both $A u t(G)$ and $\operatorname{Aut}(H)$. The greatest computational benefit is obtained if we can use $\operatorname{Aut}(G) \cap \operatorname{Aut}(H)$. The second constraint is that the automorphism group map boundary vertices, and only boundary vertices, to other boundary vertices. For the purposes of the substitution method our interest lies in connected clusters of boundary vertices, so we need to exclude group actions that move an internal vertex to the boundary (or vice versa).

\subsection{Equivalence Classes of Boundary Partitions}

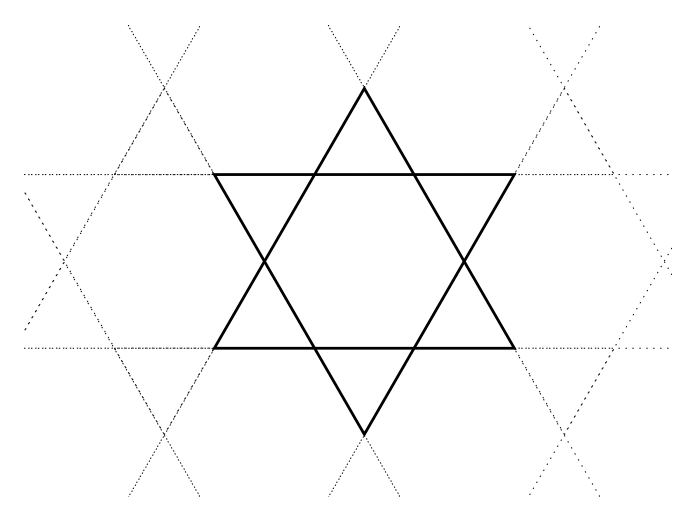

Figure 1: A Subgraph of the Kagomé Lattice

Our results are based on the fact that the actions of a group $\mathbf{G}$ (we use a bold typeface to distinguish between groups and graphs) on $G$ induce an equivalence relation on the set of bond configurations, and then, by a simple extension, on boundary partitions. The group $\mathbf{G}$ will always be $\operatorname{Aut}(G)$ or one of its 

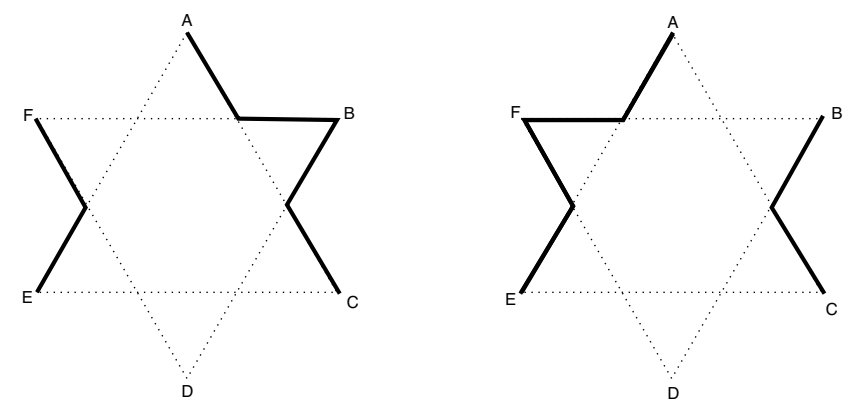

Figure 2: Bond configurations (bold edges) related by reflection through the $\overline{A D}$ axis

subgroups. In most applications of the substitution method, $G$ is planar with rotational and reflective symmetry. Thus $\mathbf{G}$ typically is a subgroup of a dihedral group.

Example 1. A portion of the infinite Kagomé lattice is shown in Figure 1. The substitution method is applied to a subgraph of the lattice, which we show with bold edges. Bond configurations are subsets of the edges of the subgraph, two instances of which are shown in Figure 2. The automorphism group for the subgraph is easily seen to be isomorphic to the dihedral group $D_{6}$, which is generated by rotations of $\pi / 3$ radians and reflections through an axis connecting opposite vertices. Thus the two bond configurations in Figure 2 are members of the same equivalence class.

Equivalent graphs under group action are said to be $\boldsymbol{G}$-equivalent. G-equivalent classes are well known to satisfy the conditions of an equivalence relation (for example, see Stanley [10]).

However, our real interest is in forming equivalence classes of boundary partitions, not bond configurations. We use the mapping from a bond configuration to a boundary partition to extend G-equivalence to boundary partitions:

Definition 1. Boundary partitions $\pi_{1}$ and $\pi_{2}$ are G-equivalent, denoted $\pi_{1} \sim \pi_{2}$, if there exist bond configurations $\kappa_{1}$ and $\kappa_{2}$ and $f \in \boldsymbol{G}$, with $\Pi\left(\kappa_{1}\right)=\pi_{1}, \Pi\left(\kappa_{2}\right)=\pi_{2}$, such that $\kappa_{1}=f\left(\kappa_{2}\right)$.

We establish some basic properties following from Definition 1.

Lemma 1. Given $\pi_{1} \sim \pi_{2}$, let $K_{1}=\left\{\kappa: \Pi(\kappa)=\pi_{1}\right\}, K_{2}=\left\{\kappa: \Pi(\kappa)=\pi_{2}\right\}$. There is a one to one correspondence between $K_{1}$ and $K_{2}$.

Proof. Since $\pi_{1} \sim \pi_{2}$ there is some $f \in \mathbf{G}$ such that $\pi_{2}=f\left(\pi_{1}\right)$. Select any $\kappa_{1} \in K_{1}$. According to Fact $4, \kappa_{1}$ maps to the unique element $\kappa_{2}=f\left(\kappa_{1}\right)$ in $K_{2}$. Moreover, from the group cancellation property, $\kappa_{2}$ maps into the unique element $\kappa_{1}=f^{-1}\left(\kappa_{2}\right)$ in $K_{1}$. Thus there is a bijection between $K_{1}$ to $K_{2}$.

The following lemma is a major reason for the usefulness of these equivalence classes:

Lemma 2. If $\pi_{1} \sim \pi_{2}$ then $P_{\pi_{1}}^{G}(p)=P_{\pi_{2}}^{G}(p)$.

Proof. By definition

$$
P_{\pi_{1}}^{G}(p)=\sum_{\kappa \in K_{1}} p^{|E(\kappa)|}(1-p)^{|E(G)|-|E(\kappa)|}
$$

and

$$
P_{\pi_{2}}^{G}(p)=\sum_{\kappa \in K_{2}} p^{|E(\kappa)|}(1-p)^{|E(G)|-|E(\kappa)|} .
$$

From Lemma 1 there is a one to one correspondence between the sets $K_{1}$ and $K_{2}$. The corresponding elements of the two sets are related by automorphisms, so by Fact 3 they have the same probability polynomial. Therefore the two sums are equal.

Another useful fact is that: 
Fact 5. If $\pi_{1} \sim \pi_{2}$, then $\pi_{1}$ and $\pi_{2}$ have the same number of clusters.

Proof. The number of connected clusters is unchanged by an automorphism action.

The following is a special case of a well known result. The proof is straightforward; for example, see Liu's [6] Theorem 5-1.

Lemma 3. The G-equivalence relation on the set of boundary partitions is an equivalence relation.

\subsection{The Class Lattice}

The sets of G-equivalent boundary partitions are the major focus of this paper, so we give them a name:

Definition 2. A boundary partition class, or simply a class, is a maximal set of $\boldsymbol{G}$-equivalent boundary partitions.

If $\pi^{\prime}$ is a representative member of class $\mathcal{C}$, then $\mathcal{C}=\left\{\pi \in \mathcal{B}: \pi \sim \pi^{\prime}\right\}$.

We will construct a partially ordered set of classes, so we need a definition of refinement that applies to classes:

Definition 3. Let $\mathcal{C}_{1}$ and $\mathcal{C}_{2}$ be equivalence classes of partitions, then class $\mathcal{C}_{1}$ is a refinement of $\mathcal{C}_{2}$, denoted $\mathcal{C}_{1} \preceq \mathcal{C}_{2}$, if for some $\pi_{1} \in \mathcal{C}_{1}$ there exists $\pi_{2} \in \mathcal{C}_{2}$ such that $\pi_{1} \leq \pi_{2}$.

The class lattice, denoted $\mathcal{P} / \mathbf{G}$, is the partially ordered set obtained from the partition lattice by lumping $\mathbf{G}$-equivalent partitions into equivalence classes. We also call $\mathcal{P} / \mathbf{G}$ the symmetry reduced lattice. We verify that:

Lemma 4. $\mathcal{P} / \boldsymbol{G}$ with refinement is a partially ordered set.

Proof. We show the three poset conditions are met for $\mathcal{P} / \mathbf{G}$. Let $\mathcal{C}_{1}, \mathcal{C}_{2}$, and $\mathcal{C}_{3}$ be boundary partition classes.

- Reflexive $(\mathcal{C} \preceq \mathcal{C}): \pi \leq \pi$ for all $\pi \in \mathcal{C}$, so $\mathcal{C} \preceq \mathcal{C}$ for all $\mathcal{C} \in \mathcal{P} / \mathbf{G}$.

- Antisymmetric $\left(\mathcal{C}_{1} \preceq \mathcal{C}_{2}\right.$ and $\mathcal{C}_{2} \preceq \mathcal{C}_{1}$ imply $\left.\mathcal{C}_{1}=\mathcal{C}_{2}\right)$ : Assume that $\pi_{1} \leq \pi_{2}$ and $\pi_{2} \leq \pi_{1}^{\prime}$, where $\pi_{1}$ and $\pi_{1}^{\prime} \in \mathcal{C}_{1}, \pi_{2} \in \mathcal{C}_{2}$. From Fact 5, partitions $\pi_{1}$ and $\pi_{1}^{\prime}$ have the same number of clusters. Furthermore $\pi_{2}$ must have the same number of clusters as $\pi_{1}$ and $\pi_{1}^{\prime}$. But $\pi_{1} \leq \pi_{2}$ where both have the same number of clusters can occur only if $\pi_{1}=\pi_{2}$.

- Transitive $\left(\mathcal{C}_{1} \preceq \mathcal{C}_{2}\right.$ and $\mathcal{C}_{2} \preceq \mathcal{C}_{3}$ imply $\left.\mathcal{C}_{1} \preceq \mathcal{C}_{3}\right)$ : By definition, for some $\pi_{1} \in \mathcal{C}_{1}$ there exists $\pi_{2} \in \mathcal{C}_{2}$ such that $\pi_{1} \leq \pi_{2}$. Likewise, for some $\pi_{2}^{\prime} \in \mathcal{C}_{2}$ there exists $\pi_{3}^{\prime} \in \mathcal{C}_{3}$ such that $\pi_{2}^{\prime} \leq \pi_{3}^{\prime}$. Since $\pi_{2}$ and $\pi_{2}^{\prime}$ are in $\mathcal{C}_{2}$ they are $\mathbf{G}$-equivalent, so there exists an automorphism $f$ where $\pi_{2}=$ $f\left(\pi_{2}^{\prime}\right)$. Then there exists an element $\pi_{3}=f\left(\pi_{3}^{\prime}\right)$ in $\mathcal{C}_{3}$, with the property that $\pi_{2} \leq \pi_{3}$. From transitivity of $\leq$ for boundary partitions, $\pi_{1} \leq \pi_{3}$. Thus $\pi_{1} \in \mathcal{C}_{1}$ is a refinement of $\pi_{3} \in \mathcal{C}_{3}$, showing $\mathcal{C}_{1} \preceq \mathcal{C}_{3}$.

Additionally, the class poset is a lattice. The class lattice is graded, of the same rank as the partition lattice from which it derives.

\section{Finding the Extremal Up-set Equation Solutions}

We have introduced symmetry reduction because, as we now show, it allows us to perform the substitution method calculation on a poset that is much smaller than $\mathcal{P}_{n}$. Recall that the substitution method calls for finding the extremal solutions to the up-set equations on the partition lattice. We show in Theorem 1 that these extremal solutions are the same as those found on the symmetry reduced lattice $\mathcal{P} / \mathbf{G}$. The computational savings due to searching the smaller poset can be considerable, often making feasible a calculation that was previously out of reach. 
We begin by introducing some notation and terminology to simplify exposition. In this section $G$ and $H$ are finite graphs with equal numbers of boundary vertices, $P_{\bullet}^{G}(p)$ and $P_{\bullet}^{H}(p)$ are the associated probability polynomials on the poset $\mathcal{P}$ of partitions of boundary vertices, and $\mathbf{G}$ is the largest subgroup of both $\operatorname{Aut}(G)$ and $\operatorname{Aut}(H)$ satisfying the constraints previously described. We write $p_{U}^{*}$ for the solution to the up-set equation for $U$, that is, $P_{U}^{G}\left(p_{U}^{*}\right)=P_{U}^{H}\left(p_{c}\right)$, where $p_{c}$ is the known critical probability for the lattice $\mathcal{H}$.

A class $\mathcal{C}$ is said to be complete in up-set $U$ if $\mathcal{C} \subseteq U$, incomplete if $\mathcal{C}$ is not complete and $\mathcal{C} \cap U \neq \emptyset$. A set of partitions $S \subseteq \mathcal{B}$ is said to be possible if there is some $p \in(0,1)$ such that $P_{S}^{G}(p)>0$ or $P_{S}^{H}(p)>0$. If $S$ is not possible then it is impossible. Note that by Fact 1 , if $S$ is possible for any value of $p$, then it is possible for all $p \in(0,1)$. Only possible sets of partitions affect the solution to the up-set equations. If $P_{U}^{G}(p)=P_{U}^{H}\left(p_{c}\right)$, and $S$ is impossible, then $P_{U \cup S}^{G}(p)=P_{U \cup S}^{H}\left(p_{c}\right)$.

The following lemma demonstrates that under the certain conditions a monotonicity result holds for a related set of up-set equation solutions. This result is crucial to proving Theorem 1.

Lemma 5. Let $U$ be a non-trivial up-set of $\mathcal{P}$, and $S$ a subset of $U$ such that $U-S$ is also an up-set. Let $S^{\prime}$ be the image of $S$ under a group action, that is, there exists $f \in \boldsymbol{G}$ such that $S^{\prime}=\{x: x=f(y), y \in$ $S\}$, such that $U \cup S^{\prime}$ is an up-set, and $U \cap S^{\prime}=\emptyset$.

Then either

$$
\begin{aligned}
p_{U \cup S^{\prime}}^{*}<p_{U}^{*}<p_{U-S}^{*} \\
p_{U \cup S^{\prime}}^{*}=p_{U}^{*}=p_{U-S}^{*} \\
\quad o r \\
p_{U \cup S^{\prime}}^{*}>p_{U}^{*}>p_{U-S}^{*}
\end{aligned}
$$

Proof. Note that we consider a non-trivial up-set to be any non-empty up-set, except that generated by the 0 partition. Since the up-set generated by the 0 partition contains all poset elements, its probability polynomial is identically equal to 1 , and the up-set equation has no unique solution.

The elements of $S$ and $S^{\prime}$ are in one to one correspondence, and corresponding partitions are members of the same class. We conclude from Lemma 2 that $S$ and $S^{\prime}$ have the same probability polynomials, which we denote $P_{S}^{G}(p)$ and $P_{S}^{H}(p)$ (respectively $P_{S^{\prime}}^{G}(p)$ and $P_{S^{\prime}}^{H}(p)$ ). The up-set equations for $U \cup S^{\prime}$, $U$, and $U-S$, respectively, are then

$$
\begin{array}{ll}
P_{U}^{G}(p)+P_{S}^{G}(p) & =P_{U}^{H}\left(p_{c}\right)+P_{S}^{H}\left(p_{c}\right) \\
P_{U}^{G}(p) & =P_{U}^{H}\left(p_{c}\right) \\
P_{U}^{G}(p)-P_{S}^{G}(p) & =P_{U}^{H}\left(p_{c}\right)-P_{S}^{H}\left(p_{c}\right) .
\end{array}
$$

By subtraction we define

$$
\begin{array}{lll}
g_{1}(p)=P_{U}^{G}(p)+P_{S}^{G}(p) & -P_{U}^{H}\left(p_{c}\right)-P_{S}^{H}\left(p_{c}\right), \\
g_{2}(p)=P_{U}^{G}(p) & -P_{U}^{H}\left(p_{c}\right), \\
g_{3}(p)=P_{U}^{G}(p)-P_{S}^{G}(p) & -P_{U}^{H}\left(p_{c}\right)+P_{S}^{H}\left(p_{c}\right),
\end{array}
$$

and

$$
k(p)=P_{S}^{G}(p)-P_{S}^{H}\left(p_{c}\right),
$$

then rewrite the up-set equations as

$$
\begin{aligned}
& g_{1}(p)=g_{2}(p)+k(p)=0 \\
& g_{2}(p)=0 \\
& g_{3}(p)=g_{2}(p)-k(p)=0 .
\end{aligned}
$$

Since each polynomial $g_{j}(p)$ is an up-set polynomial plus a constant, it is still non-decreasing. We know from Fact 2 that each up-set equation has a unique solution in $[0,1]$. Let $p_{1}^{*}, p_{2}^{*}$, and $p_{3}^{*}$ represent the solutions to Equations $1-3$, respectively. To prove the Lemma we need to show that either $p_{3}^{*}<p_{2}^{*}<$ $p_{1}^{*}, p_{3}^{*}=p_{2}^{*}=p_{1}^{*}$, or $p_{3}^{*}>p_{2}^{*}>p_{1}^{*}$. 
In fact the ordering is determined by the sign of $k\left(p_{2}^{*}\right)$. If $k\left(p_{2}^{*}\right)>0$ the polynomial values are ordered: $g_{1}\left(p_{2}^{*}\right)>g_{2}\left(p_{2}^{*}\right)=0>g_{3}\left(p_{2}^{*}\right)$. From the Intermediate Value Theorem and monotonicity we conclude that $p_{1}^{*}<p_{2}^{*}<p_{3}^{*}$. If $k\left(p_{2}^{*}\right)<0$, the opposite occurs: $p_{3}^{*}<p_{2}^{*}<p_{1}^{*}$. The last possibility is $k\left(p_{2}^{*}\right)=0$, in which case all three equations have the same solution (that is, $p_{3}^{*}=p_{2}^{*}=p_{1}^{*}$ ). This occurs if the sets $S$ and $S^{\prime}$ are impossible, or if $P_{S}^{G}\left(p_{2}^{*}\right)=P_{S}^{H}\left(p_{c}\right)$.

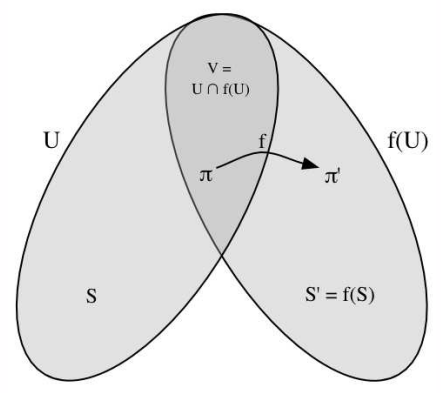

Figure 3: Sets used in the proof of Theorem 1. $\pi$ is a member of an incomplete class in $U, \pi^{\prime}$ is a member of the same class that is not contained in $U$.

Theorem 1 is the formal statement of the fact that the extremal solutions to up-set equations on the class poset equal the extremal solutions on the partition poset:

Theorem 1. There exist up-sets $U_{M}$ and $U_{m}$, which are unions of classes, such that

$$
p_{U_{M}}^{*}=\max \left\{p_{U}^{*}: U \text { is an up-set of } \mathcal{P}\right\}
$$

and

$$
p_{U_{m}}^{*}=\min \left\{p_{U}^{*}: U \text { is an up-set of } \mathcal{P}\right\}
$$

Proof. We only prove the result for $p_{U_{M}}^{*}$, since a similar argument suffices for the minimum solution.

The proof is by contradiction. Suppose that the maximum solution on the partition lattice, $p^{*}$, is not achieved by any up-set that consists entirely of complete classes. Let $U$ be a maximal up-set of $\mathcal{P}$, not necessarily unique, satisfying $p_{U}^{*}=p^{*}$, containing at least one incomplete class. Let $\mathcal{C}$ denote an incomplete class in $U$ of highest rank (that is, with the shortest length chain from $\mathcal{C}$ to the $\hat{1}$ element).

Choose a partition $\pi$ from $U \cap \mathcal{C}$, and $\pi^{\prime}$ a partition from $\mathcal{C}-U$. Since $\mathcal{C}$ is an equivalence class induced by $\mathbf{G}$ there is a group element $f$ such that $\pi^{\prime}=f(\pi)$. Form the sets $f(U)=\{x \in \mathcal{P}: x=$ $f(y), y \in U\}, V=U \cap f(U), S=U-V$, and $S^{\prime}=f(S)$. We illustrate the relationship between these sets in Figure 3. Then it is easily seen that:

- $f(U)=V \cup S^{\prime}$ is an up-set. $V \cup S^{\prime}$ is isomorphic to the up-set $U=V \cup S$, where the group element $f$ is an order-preserving bijection between the two sets.

- $U \cup S^{\prime}$ is an up-set, since $U \cup S^{\prime}=U \cup f(U)$, and the union of two up-sets is an up-set.

- The intersection of two up-sets is an up-set, so $V=U \cap f(U)$ is an up-set.

- $U \cap S^{\prime}=\emptyset$ by construction.

The conditions needed to apply Lemma 5 to $U, S$, and $S^{\prime}$ are satisfied. Therefore the up-set equation solutions are ordered in one of three ways:

$$
\begin{aligned}
& p_{U \cup S^{\prime}}^{*}<p_{U}^{*}<p_{U-S}^{*} \\
& p_{U-S}^{*}=p_{U}^{*}=p_{U \cup S^{\prime}}^{*}
\end{aligned}
$$




$$
p_{U-S}^{*}>p_{U}^{*}>p_{U \cup S^{\prime}}^{*}
$$

Equation 4 and Equation 6 cannot hold, since, by assumption, there is no up-set with solution greater than $p_{U}^{*}$. Therefore we are left with Equation 5. As noted above, $U \cup S^{\prime}$ is an up-set. Thus we can expand $U$ to obtain a larger up-set that still attains $p^{*}$. However, we assumed that $U$ was maximal, so that we have reached a contradiction.

We conclude, therefore, that the maximal up-set attaining the solution $p^{*}$ consists of complete classes of partitions.

Knowing that we can use symmetry reduction to find the extremal solutions with reduced computational cost, we want to estimate the improvement. The improvement is based on the reduction in the number of up-set equations that need to be solved. We know of no closed form result that provides this count, either exactly or asymptotically. However, in Appendix A we describe a computation to obtain the number of elements, and the number of elements by rank, for the symmetry reduced poset.

These values can then be used to bound the number of up-sets in the class lattice. There is a one to one correspondence between up-sets and antichains. Since a rank of a poset forms an antichain, the size of the power set of the largest rank is a lower bound for the number of up-set equations. Similarly, the number of up-set equations is bounded above by the size of the power set of all elements in the class lattice. In practice these bounds are very loose.

\section{Bounds on $p_{c}$ for the $\left(3,12^{2}\right)$ Lattice}

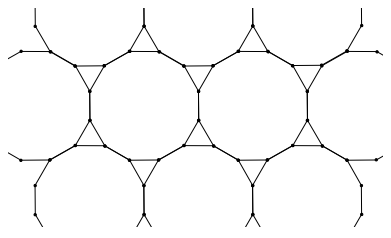

Figure 4: The $\left(3,12^{2}\right)$ Lattice

In this section we show how the theory described in this paper is used to calculate improved bounds on $p_{c}$ for a particular lattice. Our example is the "extended Kagomé" or "star" lattice, shown in Figure 4. It is one of the 11 Archimedean lattices, that is, vertex transitive tilings of the plane by regular polygons. Since the lattice is vertex transitive it can be characterized by listing the number of sides in each $n$-gon adjacent to any given vertex. In this example each vertex is adjacent to a triangle and 2 12-gons. Thus this lattice is also known as the $\left(3,12^{2}\right)$ lattice .

There is a long standing conjecture by Tsallis [13] that the exact critical probability for the $\left(3,12^{2}\right)$ lattice is $.739830 \ldots$... Wierman [18] has previously established rigorous bounds of $(0.738598,0.744900)$ using the substitution method.
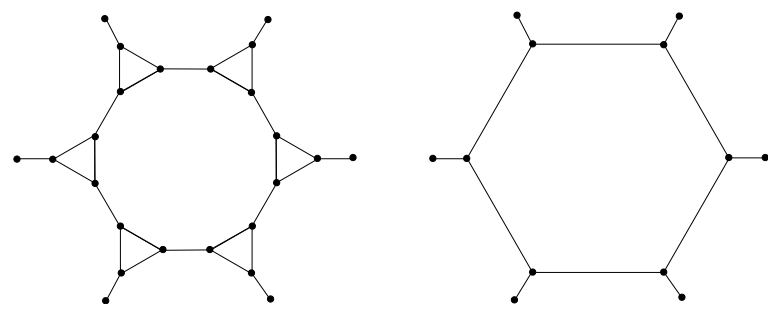

Figure 5: The $\left(3,12^{2}\right)$ Substitution Region: Unsolved vs. Solved Subgraph 
To apply the substitution method we need to decompose the $\left(3,12^{2}\right)$ lattice into isomorphic, edgedisjoint subgraphs, and substitute alternative subgraphs in order to obtain a lattice which is exactly solved. Our substitution region is the subgraph shown on the left in Figure 5. It consists of a single 12-gon, the 6 triangles on the corners, and 6 half edges. The half edges are needed so that the lattice decomposition is edge-disjoint. If the full edges are open with probability $p$ then the half edges are open with probability $\sqrt{p}$. When we substitute a hexagon plus six half-edges for this subgraph, as shown on the right in Figure 5, we obtain the hexagonal lattice, which has known critical probability $1-2 \sin (\pi / 18)$ (Wierman [14]).

Both substitution regions have 6 boundary vertices, so the relevant partition lattice is $\mathcal{P}_{6}$. The cardinality of $\mathcal{P}_{6}$ is the $6^{\text {th }}$ Bell number, 203. It is easily shown that the largest rank of $\mathcal{P}_{6}$ has 90 elements. While the number of up-sets is unknown, the cardinality of the power set of the largest rank, that is, $2^{90} \approx 10^{27}$ is a lower bound. Thus searching the entire lattice to determine the extremal solutions would require solving at least $10^{27}$ up-set equations. This is not practical.

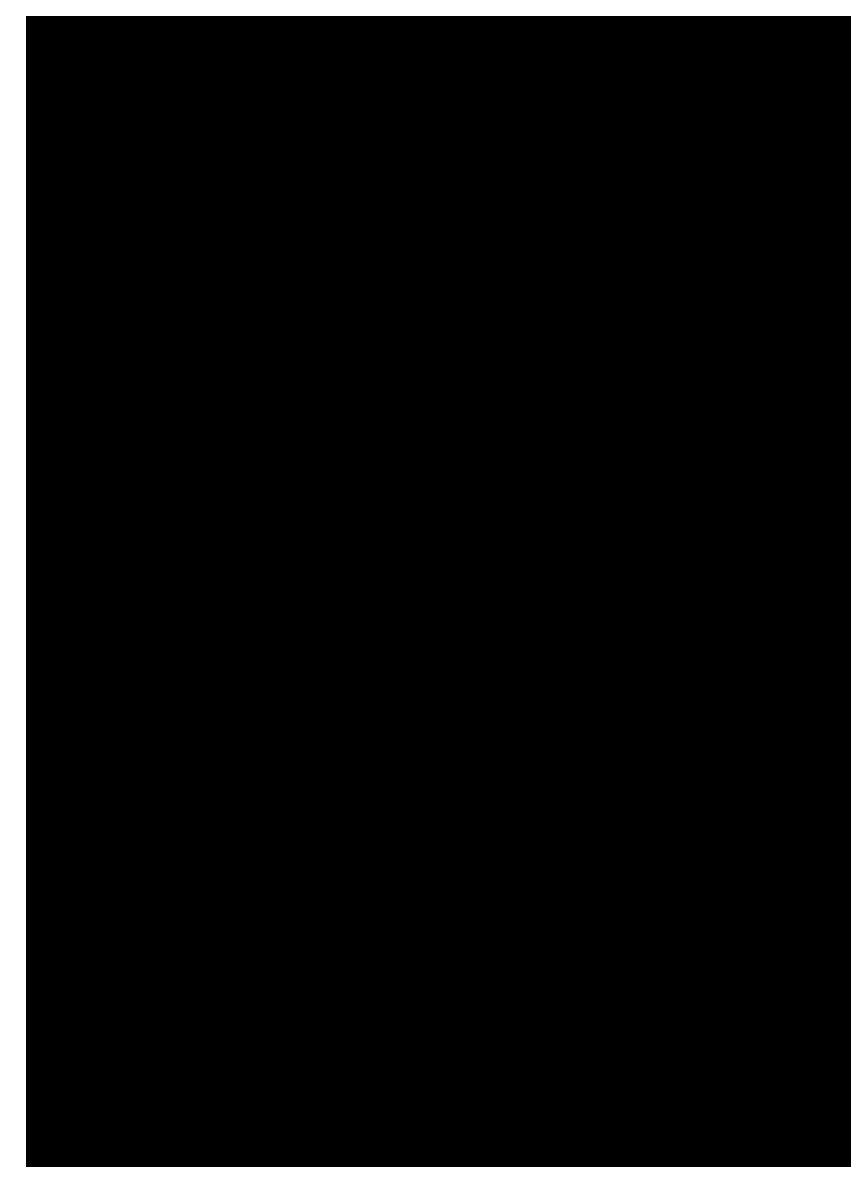

Figure 6: Hasse diagram for the class lattice $\mathcal{P}_{6} / D_{6}$. Symmetry reduction has reduced the poset from 203 elements to the 37 shown here, and from at least $10^{27}$ up-sets to 95,708 up-sets.

Due to their symmetry, the appropriate automorphism group for both these graphs is the dihedral group $D_{6} . D_{6}$ also satisfies the constraint that boundary vertices map only to boundary vertices. When we apply the $D_{6}$ group actions to $\mathcal{P}_{6}$ we obtain a class lattice containing 37 elements (see Appendix A). The Hasse diagram for the class lattice is shown in Figure 6. While the partition lattice had greater than $10^{27}$ up-set equations to solve, we find that the class lattice has precisely 95,708 up-set equations to solve. This is a small enough number that finding the extremal solutions by solving all up-set equations is easily accomplished by a computer.

To summarize, the complete computational procedure is the following: 
1. Determine the automorphism groups for $G$ and $H$, satisfying the constraints. In this example, the appropriate group is clearly $D_{6}$. If there is any doubt, the program nauty (see McKay [7]) can be used.

2. Using the automorphism group, define the class lattice by classifying the boundary partitions and determining the inclusion relationships. We have developed a computer program called class ify to perform this calculation.

3. Enumerate the up-sets for $\mathcal{P} / \mathbf{G}$ calculated in the step 2. We used the algorithm from Squires [8] that performs a Hamiltonian walk on the set of up-sets.

4. Calculate the $\pi$-polynomials for all equivalence classes of boundary vertices.

5. Search for the extremal up-set equation solutions among the set of all up-sets. Using Newton's method on a PowerPC processor, this only took about 20 minutes. We note that this problem is especially favorable for Newton's method. A solution is guaranteed to exist in $(0,1)$ and it is unique in that interval. We use the mid-point of Wierman's [18] previous bounds as the starting point for the Newton iteration, insuring that the starting point is quite close to the up-set equation solution.

The minimum and maximum solutions, 0.739399 and 0.741757 , correspond to the up-sets $A B C D E F$ and everything but $A|B| C|D| E \mid F$. Note that the interval between our bounds includes Tsallis's conjectured value $0.739830 \ldots$, although the lower bound comes close to excluding it.

\section{A Enumerating the Class Poset}

In this appendix we describe a method for calculating the number of elements in the symmetry reduced poset. We have found this calculation useful for estimating the work required to solve specific problems and for checking computer results. In the interests of brevity we won't review the theory underlying these techniques. The reader who is unfamiliar with the theory can refer to Liu [6] or Beckenbach [1] for a complete discussion.

We introduce a different representation for a boundary partition, called the class vector. The class vector assigns an integer to each set of connected boundary vertices. For a partition of $A, B, \ldots, F$ the class vector is of length 6 , and for each $i=1, \ldots, 6$ the $i$ th entry is the class (set of connected vertices) to which the $i$ th vertex belongs. For example, the class vector for boundary partition $A B|C D| E F$ is 112233. The class vector representation reveals that partitioning the boundary vertices can be seen as a coloring problem, with each "color" representing a cluster of connected boundary vertices. Note that the actual color assigned to a boundary vertex does not matter, only the set of partitions sharing the same color. That is, partition 112233 is considered equivalent to 221133 and 223311.

Counting colorings of a set under group action is a classic application of Pólya's Enumeration Theorem. However Pólya's Theorem doesn't go quite far enough. It gives us a method to count all distinct colorings. We need a method that also incorporates the notion that "distinct clusters are colored differently, but we don't care what the color is."

De Bruijn's Theorem is a generalization of Pólya's Theorem that allows us to count equivalence classes in precisely this way. Two groups are employed. The first group, $G$, represents the geometric symmetries of the underlying graph; while the second, $H$, represents a notion of what constitute equivalent colorings.

In order to apply de Bruijn's Theorem we need to know the cycle index polynomial for each group. The permutations that comprise $G$ and $H$ can be categorized by the number of cyclic permutations of each length. If permutation $\pi$ has $b_{1}$ cycles of length $1, b_{2}$ of length $2, \ldots, b_{k}$ cycles of length $k$, then we use the monomial $x_{1}^{b_{1}} x_{2}^{b_{2}} \cdots x_{k}^{b_{k}}$ to to represent the number of cycles of each length in $\pi$. This is called the cycle structure representation of $\pi$. Given a group $G$, the cycle index polynomial $Z_{G}$ is the sum of the cycle structure representations of the permutations of $G$, divided by the number of permutations in $G$ :

$$
Z_{G}=\frac{1}{|G|} \sum_{\pi \in G} x_{1}^{b_{1}} x_{2}^{b_{2}} \cdots x_{k}^{b_{k}}
$$

Formally de Bruijn's Theorem states: 
Theorem 2. $X$ is a set, $C$ a set of colors, $Z_{G}$ and $Z_{H}$ are the cycle index polynomials for $G$ and $H$. Then the number of equivalence classes of functions from $X$ to $C$ is the value of the expression

$$
Z_{G}\left(\frac{\partial}{\partial z_{1}}, \frac{\partial}{\partial z_{2}}, \frac{\partial}{\partial z_{3}}, \ldots\right) \times\left. Z_{H}\left[e^{z_{1}+z_{2}+z_{3}+\cdots}, e^{2\left(z_{1}+z_{2}+z_{3}+\cdots\right)}, e^{3\left(z_{1}+z_{2}+z_{3}+\cdots\right)}, \ldots\right]\right|_{z_{1}=z_{2}=\cdots=0} .
$$

Liu [6] provides a proof of this theorem.

We illustrate its applicability to our problem with two examples.

Example 2. We wish to count the elements of $\mathcal{P}_{6}$ reduced by hexagonal symmetry. Hexagonal symmetry suggests that we use the dihedral group $D_{6}$ for $G$ in de Bruijn's Theorem. The notion that the actual color assignments don't matter is equivalent to applying the symmetric group to the colors. Any permutation of the colors results in an equivalent "coloring". Therefore we need the cycle index polynomials for $D_{6}$ and for the symmetric group $S_{6}$. These are

$$
Z_{D_{6}}\left(z_{1}, z_{2}, z_{3}, z_{6}\right)=\frac{1}{12}\left(z_{1}^{6}+4 z_{2}^{3}+2 z_{3}^{2}+3 z_{1}^{2} z_{2}^{2}+2 z_{6}\right)
$$

and

$$
\begin{aligned}
Z_{S_{6}}\left(z_{1}, z_{2}, z_{3}, z_{4}, z_{5}, z_{6}\right)=\frac{1}{720}\left(z_{1}^{6}+\right. & 15 z_{1}^{4} z_{2}+40 z_{1}^{3} z_{3}+90 z_{1}^{2} z_{4}+144 z_{1} z_{5} \\
& \left.+45 z_{1}^{2} z_{2}^{2}+15 z_{2}^{3}+120 z_{1} z_{2} z_{3}+90 z_{2} z_{4}+40 z_{3}^{2}+120 z_{6}\right) .
\end{aligned}
$$

Using de Bruijn's Theorem with $Z_{G}=Z_{D_{6}}$ and $Z_{H}=Z_{S_{6}}$, and after a very tedious calculation, we get the number 37 . The use of hexagonal symmetry has reduced the poset size from its original 203 elements to a class lattice of 37 elements.

Example 3. By a small modification of the technique we can count the elements for each rank in the class lattice. We first apply $Z_{S_{1}}$ to find the number of elements in the class with 1 cluster. This is, of course, always 1 . Then we apply $Z_{S_{2}}$ to find the number of elements in the class with 1 or 2 clusters. By subtracting the number of 1 cluster classes from this result we find the number of 2 cluster classes. We iterate this calculation, that is, calculate the number of classes using $Z_{S_{k}}$, subtract the number of $1, \ldots, k-1$ cluster classes to get the number of $k$ cluster classes. We perform this calculation for $k=2, \ldots, n$.

Table 1 shows the results of calculating these values for $n$-gons from 1 to 12 vertices, assuming dihedral group symmetry to reduce the partition lattice.

\begin{tabular}{|rrrrrrrrrrrrrrr|}
\hline & & & \multicolumn{1}{c}{ Number of Clusters } & \multicolumn{1}{c|}{ Total } & \multicolumn{1}{c|}{ Total } \\
$n$-gon & 1 & 2 & 3 & 4 & 5 & 6 & 7 & 8 & 9 & 10 & 11 & 12 & Classes & Partitions \\
\hline 1 & 1 & - & - & - & - & - & - & - & - & - & - & - & 1 & 1 \\
\hline 2 & 1 & 1 & - & - & - & - & - & - & - & - & - & - & 2 & 2 \\
\hline 3 & 1 & 1 & 1 & - & - & - & - & - & - & - & - & - & 3 & 5 \\
\hline 4 & 1 & 3 & 2 & 1 & - & - & - & - & - & - & - & - & 7 & 15 \\
\hline 5 & 1 & 3 & 5 & 2 & 1 & - & - & - & - & - & - & - & 12 & 52 \\
\hline 6 & 1 & 7 & 14 & 11 & 3 & 1 & - & - & - & - & - & - & 37 & 203 \\
\hline 7 & 1 & 8 & 31 & 33 & 16 & 3 & 1 & - & - & - & - & - & 93 & 877 \\
\hline 8 & 1 & 17 & 82 & 137 & 85 & 27 & 4 & 1 & - & - & - & - & 354 & 4140 \\
\hline 9 & 1 & 22 & 202 & 478 & 434 & 171 & 37 & 4 & 1 & - & - & - & 1350 & 21147 \\
\hline 10 & 1 & 43 & 538 & 1851 & 2271 & 1249 & 338 & 54 & 5 & 1 & - & - & 6351 & 115975 \\
\hline 11 & 1 & 62 & 1401 & 6845 & 11530 & 8389 & 3056 & 590 & 70 & 5 & 1 & - & 31950 & 678570 \\
\hline 12 & 1 & 121 & 3838 & 26148 & 58400 & 56079 & 26696 & 6907 & 1014 & 96 & 6 & 1 & 179307 & 4213597 \\
\hline
\end{tabular}

Table 1: Class Count for Each Rank, for $n$-gons with Dihedral Symmetry 


\section{References}

[1] Beckenbach, E. (1981) Applied Combinatorial Mathematics, Krieger Publishing Company.

[2] Biggs, N. (1974) Algebraic Graph Theory, Cambridge University Press.

[3] Broadbent, S. R. and Hammersley, J. M. (1957) Percolation Processes. I. Crystals and mazes, Proc. Camb. Phil. Soc. 53, 629-641.

[4] Jensen, I., Guttmann A. J. and Enting I. G. (1997) The Potts model on Kagomé and honeycomb lattices, J. Phys. A: Math. Gen. 30, 8067-8083.

[5] Lindvall, T. (1992) Lectures on the Coupling Method, John Wiley \& Sons.

[6] Liu, C. L. (1968) Introduction to Combinatorial Mathematics, McGraw-Hill Inc.

[7] Mackay, B. D. (1990) nauty User's Guide (Version 1.5), Technical Report TR-CS-90-02, Department of Computer Science, Australian National University.

[8] Squires, M. B. (1994) Gray Codes and Efficient Generation of Combinatorial Structures, Ph.D. Thesis, North Carolina State University.

[9] Stanley, R. P. (1986) Enumerative Combinatorics Volume 1, Cambridge University Press.

[10] Stanley, R. P. (2000) Topics in Algebraic Combinatorics, Course notes for Mathematics 192, Harvard University.

[11] Strassen, V. (1965) The existence of probability measures with given marginals, Annals of Mathematical Statistics 36, 423-439.

[12] Sykes, M. F. and Essam, J. W. (1964) Exact critical percolation probabilities for site and bond problems in two dimensions. Journal of Mathematical Physics 5, 1117-1127.

[13] Tsallis, C. (1982) Phase Diagram of anisotropic planar Potts ferromagnets: a new conjecture. Journal of Physics C: Solid State Physics 15, L757-L764.

[14] Wierman, J. C. (1981) Bond Percolation on Honeycomb and Triangular Lattices, Advances in Applied Probability 13, 298-313.

[15] Wierman, J. C. (1989) Bond Percolation Critical Probability Bounds for the Kagomé Lattice by a Substitution Method, Disorder in Physical Systems, Grimmett G. and Welsh D.J.A. (eds.) 349-360.

[16] Wierman, J. C. (1995) Substitution Method Critical Probability Bounds for the Square Lattice Site Percolation Model, Combinatorics, Probability and Computing 4, 181-188.

[17] Wierman, J. C. (2002) An Improved Upper Bound for the Hexagonal Lattice Site Percolation Critical Probability, Combinatorics, Probability and Computing, 11, 629-643.

[18] Wierman, J. C. (2002) Bond Percolation Critical Probability Bounds for Three Archimedean Lattices, Random Structures and Algorithms 20, 508-518.

[19] Wierman, J. C. (2003) Upper and Lower Bounds for the Kagomé Lattice Bond Percolation Critical Probability, Combinatorics, Probability and Computing, 12, 95-111.

[20] Ziff, R. M., and Suding P. N. (1997) Determination of the bond percolation threshold for the Kagomé lattice, J. Phys. A: Math. Gen., 30, 5351-5359. 